\title{
Modified virtual semi-circle path planning
}

\begin{abstract}
The challenging part of path planning for an Unmanned Ground Vehicle (UGV) is to conduct a reactive navigation. Reactive navigation is implemented to the sensor based UGV. The UGV defined the environment by collecting the information to construct it path planning. The UGV in this research is known as Mobile Guard UGV-Truck for Surveillance (MG-TruckS). Modified Virtual Semi Circle (MVSC) helps the MG-TruckS to reach it predetermined goal point successfully without any collision. MVSC is divided into two phases which are obstacles detection phase and obstacles avoidance phase to compute an optimal path planning. MVSC produces shorter path length, smoothness of velocity and reach it predetermined goal point successfully.
\end{abstract}

Keyword: Obstacles avoidance; Obstacles detection; Optimal path planning; Path planning; Reactive navigation; Unmanned ground vehicle 